\begin{tabular}{c} 
Volume and Issues Obtainable at Center for Sustainability Research and Consultancy \\
Journal of Accounting and Finance in Emerging Economies \\
ISSN: 2519-0318 ISSN (E) 2518-8488 \\
Volume 7: Issue 1 March 2021 \\
CSRC \\
Journal homepage: $\underline{\text { ww.publishing.globalcsrc.org/jafee }}$ \\
\hline
\end{tabular}

\title{
Comprehensive Analysis of Tariff Barriers Worldwide: A Composite Assessment Approach
}

\author{
${ }^{1}$ Muhammad Khalid Rashid, ${ }^{2}$ Abdul Basit, ${ }^{3}$ Tehmina Fiaz Qazi, ${ }^{4}$ Abdul Aziz Khan Niazi \\ ${ }^{1}$ Govt. College of Science, Lahore, Pakistan, Krkhan55@hotmail.com \\ ${ }^{2}$ Lahore Institute of Science \& Technology, Lahore, Pakistan, abasit_shahbaz@yahoo.com \\ ${ }^{3}$ Hailey College of Banking and Finance, University of the Punjab, Lahore, Pakistan, \\ tehmina.qazi@gmail.com
}

${ }^{4}$ Institute of Business and Management, University of Engineering and Technology, Lahore, Pakistan, azizniazi@uet.edu.pk

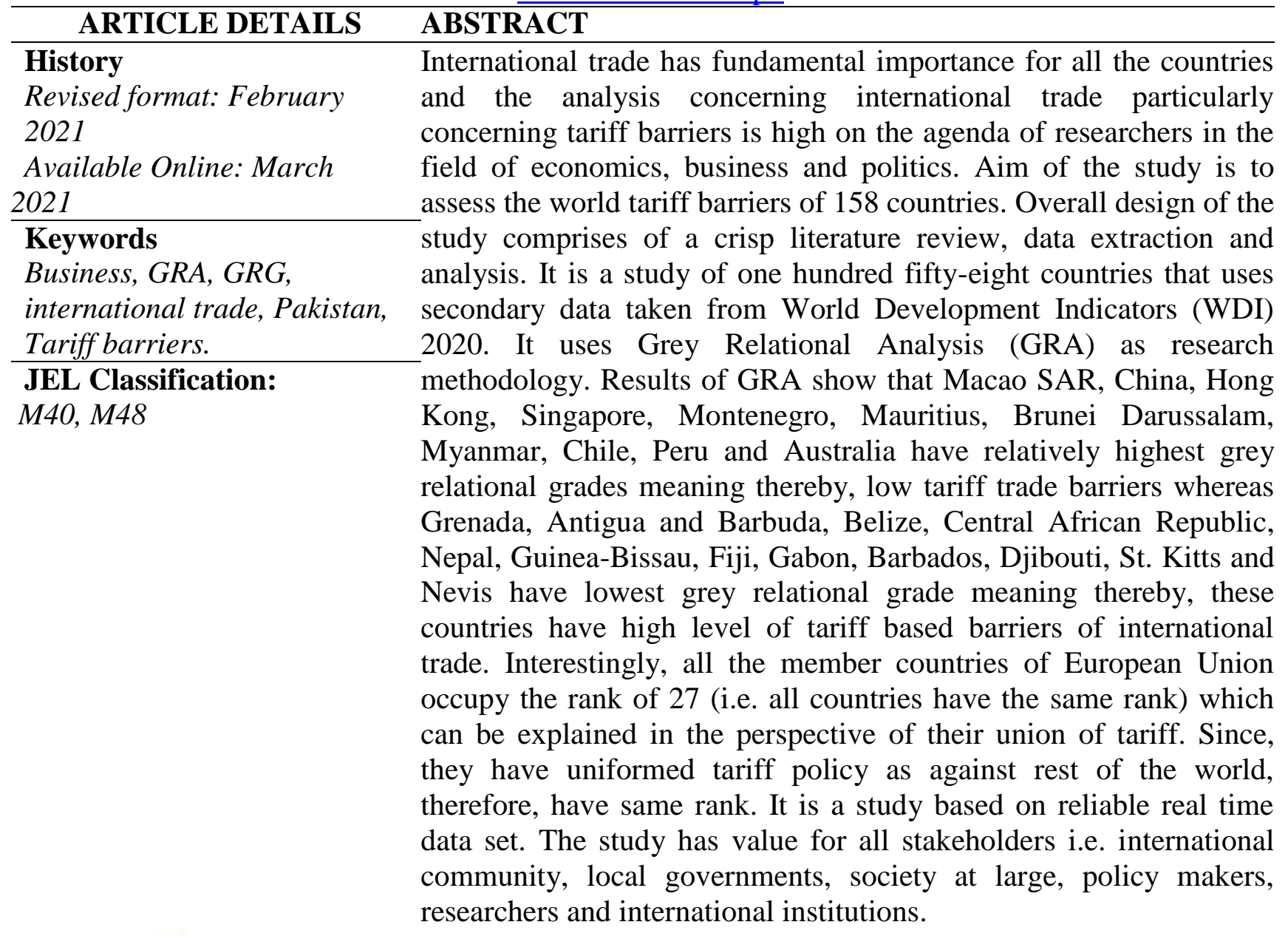

\section{OPEN ACCESS}

(C) 2020 The authors, under a Creative Commons AttributionNonCommercial 4.0

Corresponding author's email address: abasit_shahbaz@ yahoo.com

Recommended citation: Rashid, M. K., Basit, A., Qazi, T. F. \& Niazi, A. A. K. (2021). Comprehensive Analysis of Tariff Barriers Worldwide: A Composite Assessment Approach. Journal of Accounting and Finance in Emerging Economies, 7(1), 205-215 


\section{Introduction}

In the era of globalization tariff and non-tariff barriers are being removed by most of the countries in general and at bloc level in particular. Certain groups of countries have been very successful in this regard e.g. European Union. Pinpointing and assessing the barriers in international trade at country levels is high on the agenda of main stream economists. Country level comparisons are also fertile topics being investigated by contemporary researchers. It is important and research worthy for effectiveness of coordinated improvement to facilitate cross border trade. Avalanche of published literature is available in the area viz non-tariff barriers in Syria (Chemingui \& Dessus, 2008), trade liberalization and competition in China (Deng et al., 2020), trade policy of Uruguay (Estrades \& Flores, 2020), challenges of non-tariff barriers in Japanese Market (Maguire, 2001), tariff \& non-tariff barriers and examine of inequality and trade policy of India (Ural Marchand, 2019), challenges of regulating water and sanitation tariff in Brazil (Sampaio \& Sampaio, 2020), non-tariff barriers on chicken import in Russia (Soon \& Thompson, 2020), income inequality and tariff reform in Indonesia (Vadila \& Resosudarmo, 2020), China's tariffs on U.S. hardware product (Zhang et al., 2020). From the context stated in the literature it can be learned that country level assessment of tariff related barriers is imperative to provide insights to the stake holders. It is an ever green issue for research. Particularly the composite assessment of tariff barriers is call of the day. Objective of the research is therefore to assess, rank and discuss position of 158 countries on tariff related trade barriers. The study has been built on mathematical methodology opted from wide array of methodological choices viz ANP, FANP, DEMATEL, AHP, PROMETHEE, TOPSIS, DEA, GRA, VIKOR, SWARA, ISM, TISM, MICMAC, FMICMAC, IPA, ELECTRE, NSGAII, MADM, MAGDM, ARAS-F, FMEA-Model, ELECTRE-III, COPRAS-G, MULTI-MOORA, ARAS, WASPAS, MOORA, LP, IRP, SEM and Regression. To be more specific the study uses Grey Relational Analysis (GRA) as technique of data analysis. GRA is the most popular and applied part of grey systems theory. Rest of the paper is arranged as literature review, methodology, analysis \& discussion and conclusion.

\section{Literature Review}

The problem under investigation is espoused in the domain of economics in general and in international trade in particular. The literature of economics in the context of international trade is filthy rich. It is pertinent to set out the context of the study to account for the relevant contemporary literature. In this context, we explored wide range of literature from within the renowned data bases e.g. ScienceDiret, Emerald, Wiley Blackwell, Taylor\&Francis, JStor, Ebsco Host, etc. Apart from the general literature on international trade, while exploring the literature, researchers found numerous studies carried in the context of tariff and non-tariff barriers (Chemingui \& Dessus, 2008; Cheong \& Tang, 2018; Daly et al., 2000; DaSilva-Glasgow, 2020; Fugazza \& Maur, 2008; Grundke \& Moser, 2019; Imbruno, 2016; Jørgensen \& Schröder, 2003; Juust et al., 2020; Knobel et al., 2019; Liu et al., 2019; Magee et al., 2019; Maguire, 2001; Manzoor et al., 2019; Niu et al., 2018; Okumura, 2015; Santeramo \& Lamonaca, 2019; Schuenemann \& Kerr, 2019; Soon \& Thompson, 2020; Toshimitsu, 2008; Winchester, 2009). Aisbett and Silberberger (2020) found that countries where tariff liberalization is higher, producers can adopt relatively cheaper standard and perform at top of the list. Cambini and Soroush (2019) proposed a grid tariffs to account for costs under mechanism of net metering and concluded that employing a multi-part tariffs (variable component that imitates net effect on operating cost together with fixed component that imitates cyclical grid-connection cost of distribution networks) there is possibility to mitigate the snags of optimize prosumption and net metering. Cary (2020) uncovered that tariffs implementation invoke inefficiency, furthermore it has no effect on carbon dioxide emissions but has direct positive relationship with carbon intensities. Giammetti (2020) bolstered that Brexit costs not only for UK but also for many Europeans countries, at worst, the 0.28 and $0.5 \%$ of value added respectively and allowed countries and sectors to partially substitute the foreign products. Hayakawa et al. (2020) analyzed the relationship of import-tariff and export expansions and found a direct positive relationship between them. The study further reported that $1 \%$ decrease in importers' tariff escalate $0.8 \%$ import freight rates; reduce around $1.1 \%$ export freight rates and increase export by $0.6 \%$ to $1 \%$. He et al. (2019) affirmed that optimal 
tariffs significantly decrease by $26 \%$ globally on average after trade imbalance which is advantageous to the global trade liberalization. Juust et al. (2020) documented that confiscation of non-tariff industry specific barrier played a pertinent role in expediting European automotive exports. Kang and Dagli (2018) argued that high tariff has significant negative direct and indirect impact on trade. It is further highlighted that indirect negative effect has bigger impact than direct impact. Knobel et al. (2019) carried a comprehensive empirical study and bolstered that decrease in non-tariff barriers and decrease in time in trade costs of goods result in significant improvement in welfare gains as $\%$ of consumption of $1.7 \%$ for Kazakhstan, $4.8 \%$ for Armenia, 7.2\% for Belarus and 3.6\% for Russia. Nawaz (2019) proposed a dynamic model that originates an optimal tariff for specific import to curtail the efficiency losses during adjustment procedure. Ortiz Valverde and Latorre (2020) stated that removal of UK's tariff, advent of MFN tariffs and removal of restriction to migration broadly impacts on micro and macroeconomic of UK, China, US, EU and rest of the world. Slany (2019) revealed a significant negative impact of trade policies on foreign value added of charged tariff and positive correlated with telecommunication infrastructure. Tovar (2019) asserted that there is evident of tariff complementarity i) consumption rises, ii) greater reduction in import protection and iii) fall in imports from rest of the world. Vadila and Resosudarmo (2020) investigated that provinces having acquaintance to tariff liberalization experiencing relatively lesser income inequality, the study further highlighted that tariff cuts cause in decrease in poverty reduction. From the representation of the literature it is evident that there is enormous dispersed literature directly and indirectly related to the trade barriers. However, there is hardly any study that directly addresses the issue at global level. In order to specify the issue, it is not out of context to postulate the theoretical framework of the study.

\section{Theoretical Framework}

The study follows theoretical framework as used in World Development Indicators (WDI-2020) for representation of data set. Since, the study has adopted the data set from WDI, therefore, the variables specifications, unit of measurement and the definitions have also been adopted from WDI (Table 1). However, the variables as specified have been explored and aligned with the existing literature. A brief representation of literature concerning the variables is herein accounted for. Suwanprasert (2020) asserted that trade restrictiveness index of non-tariff barriers can be achieved weighted average mean of non-tariff barriers and it is further claimed that aggregated of non-tariff barriers can exactly be measured unlike other indexes having persistent measurement errors. Ino and Miyaoka (2020) affirmed that in case of homogenous products, higher domestic welfare is evident than import tariff to maximize profitability of domestic industry; and in case of differentiated products production control mechanism is fluctuated depending upon the variability of the products. The study further argued that government has control over domestic production of foreign firms particularly for homogenous products. Kuenzel (2020) found a significant inverse relationship between tariff overhangs and trade policy activity. Different share of tariff lines result in alternate outcome; like lower tariff overhangs compel countries to increase MostFavored Nation (MFN) applied rates without consideration of past tariff independent changes and legal ramifications. Lichtenberg and Olson (2020) buttressed that tariff rates put insignificant effects on anticipated intrusive pest introductions facing presently positive tariffs. It is further proclaimed that different tariff lines share may result in lower consumer welfare and weaken United States trade policy.

Table 1: Specification of Variables

\begin{tabular}{clcc}
\hline Code & \multicolumn{1}{c}{ Variable } & Measure & \multicolumn{1}{c}{ Criteria } \\
\hline 1 & All Products Binding Coverage & \% MRV & Smaller is Best \\
2 & All Products Weighted Mean tariff & $\%$ MRV & Smaller is Best \\
3 & All Products Share of Tariff lines with International Peaks & $\%$ MRV & Smaller is Best \\
4 & All Products Share of Tariff lines with Specific Rates & $\%$ MRV & Smaller is Best \\
5 & Primary Products Weighted Mean Tariff & $\%$ MRV & Smaller is Best \\
6 & Manufactured Products Weighted Mean Tariff & $\%$ MRV & Smaller is Best \\
\hline
\end{tabular}

The variables have been selected from the website of WDI on the basis of availability of the data and relevance to the phenomenon under investigation. Total six variables (Table 1) qualify for inclusion in 
investigation. All variables possess the characteristic "smaller is best".

\section{Methodology}

This quantitative research study follows positivism as research philosophy and deduction as research approach. It is a study of 158 countries using the secondary data extracted from the website World Development Indicators (WDI) 2020. Overall design of the study entails on review of literature, data elicitation and analysis. Traditional procedure of Grey Relational Analysis (GRA) has been applied to the data as a technique of analysis. GRA is unique methodology that has the capability to accommodate wide range of alternatives with multitude of different criteria. The scheme of symbols and the procedure has been adopted from Ertugrul et al. (2016); Qazi et al. (2021) and Niazi et al. (2021).

\subsection{Grey Relational Analysis}

Step 1: Created a data set and established decision matrix Equation 1.

$$
x_{i}(k)=\left[\begin{array}{ccc}
x_{1}(1) x_{1}(2) & \cdots & x_{1}(m) \\
\vdots & \ddots & \vdots \\
x_{n}(1) x_{n}(2) & \cdots & x_{n}(m)
\end{array}\right] \quad \text { Equation } 1
$$

Table 2: Tariff Data of Countries

\begin{tabular}{|c|c|c|c|c|c|c|c|}
\hline Sr. & Country & 1 & 2 & 3 & 4 & 5 & 6 \\
\hline 1 & Albania & 100 & 0.9 & 6.84 & 0 & 1.3 & 0.6 \\
\hline 2 & Angola & 100 & 9.4 & 21.9 & 0 & 18 & 7.1 \\
\hline$\cdots$ & $\cdots \cdots \cdots$ & $\cdots$ & $\cdots$ & $\cdots$ & $\cdots$ & $\cdots$ & $\cdots$ \\
\hline$\cdots$ & ........... & ... & ... & $\ldots$ & ... & $\ldots$ & ... \\
\hline 109 & Pakistan & 99 & 10 & 49.4 & 0 & 7.7 & 12 \\
\hline 110 & Panama & 94 & 5.4 & 0.62 & 0.1 & 14 & 5.1 \\
\hline$\cdots$ & .......... & .. & ... & $\cdots$ & $\cdots$ & ... & ... \\
\hline$\ldots$ & ........... & ... & ... & $\ldots$ & ... & ... & $\ldots$ \\
\hline 157 & Zambia & 18 & 6.2 & 27.1 & 0 & 4 & 7.4 \\
\hline 158 & Zimbabwe & 24 & 5 & 18.9 & 6 & 5.5 & 4.5 \\
\hline
\end{tabular}

Step 2: Created reference series and comparison matrix Equation 2

$$
x_{0}=\left[x_{0}(k) \ldots \ldots \ldots \ldots x_{0}(n)\right] \quad \text { Equation } 2
$$

\begin{tabular}{|c|c|c|c|c|c|c|c|}
\hline Sr. & Country & 1 & 2 & 3 & 4 & 5 & 6 \\
\hline $\mathbf{0}$ & Reference Sequence & 0 & 0 & 0 & 0 & 0 & 0 \\
\hline $\mathbf{1}$ & Albania & 100 & 0.9 & 6.84 & 0 & 1.3 & 0.6 \\
\hline 2 & Angola & 100 & 9.4 & 21.9 & 0 & 18 & 7.1 \\
\hline$\cdots$ & n.......... & $\cdots$ & $\cdots$ & $\cdots$ & $\cdots$ & $\cdots$ & $\cdots$ \\
\hline$\ldots$ & n........ & ... & ... & $\ldots$ & .. & $\cdots$ & $\cdots$ \\
\hline 109 & Pakistan & 99 & 10 & 49.4 & 0 & 7.7 & 12 \\
\hline 110 & Panama & 94 & 5.4 & 0.62 & 0.1 & 14 & 5.1 \\
\hline$\cdots$ & ........... & $\cdots$ & $\cdots$ & $\cdots$ & $\cdots$ & $\cdots$ & $\cdots$ \\
\hline$\ldots$ & (n....... & ... & ... & $\ldots$ & ... & ... & $\ldots$ \\
\hline 157 & Zambia & 18 & 6.2 & 27.1 & 0 & 4 & 7.4 \\
\hline 158 & Zimbabwe & 24 & 5 & 18.9 & 6 & 5.5 & 4.5 \\
\hline
\end{tabular}

Table 3: Reference Sequence and Comparable Sequences

Step 3: Created a normalized matrix Equation 3 and prepare Table 3:

Smaller the best $\quad x_{i}(k)=\frac{\max x_{i}^{(o)}(k)-x_{i}^{(0)}(k)}{\max x_{i}^{(0)}(k)-\min x_{i}^{(0)}(k)} \quad$ Equation 3

Table 4: Normalized Comparable Sequences 


\begin{tabular}{clcccccc}
\hline $\mathbf{0}$ & Reference Sequence & 1.0000 & 1.0000 & 1.0000 & 1.0000 & 1.0000 & 1.0000 \\
$\mathbf{1}$ & Albania & 0.0000 & 0.9571 & 0.9055 & 1.0000 & 0.9658 & 0.9700 \\
$\mathbf{2}$ & Angola & 0.0000 & 0.5524 & 0.6975 & 1.0000 & 0.5263 & 0.6450 \\
$\ldots$ & $\ldots \ldots \ldots \ldots$. & $\ldots$ & $\ldots$ & $\ldots$ & $\ldots$ & $\ldots$ & $\ldots$ \\
$\ldots$ & $\ldots \ldots \ldots .$. & $\ldots$ & $\ldots$ & $\ldots$ & $\ldots$ & $\ldots$ & $\ldots$ \\
$\mathbf{1 0 9}$ & Pakistan & 0.0100 & 0.5238 & 0.3177 & 1.0000 & 0.7974 & 0.4000 \\
$\mathbf{1 1 0}$ & Panama & 0.0600 & 0.7429 & 0.9914 & 0.9872 & 0.6316 & 0.7450 \\
$\ldots$ & $\ldots \ldots \ldots \ldots$. & $\ldots$ & $\ldots$ & $\ldots$ & $\ldots$ & $\ldots$ & $\ldots$ \\
$\ldots$ & $\ldots \ldots . . .$. & $\ldots$ & $\ldots$ & $\ldots$ & $\ldots$ & $\ldots$ & $\ldots$ \\
$\mathbf{1 5 7}$ & Zambia & 0.8200 & 0.7048 & 0.6257 & 1.0000 & 0.8947 & 0.6300 \\
$\mathbf{1 5 8}$ & Zimbabwe & 0.7600 & 0.7619 & 0.7390 & 0.2308 & 0.8553 & 0.7750 \\
\hline
\end{tabular}

For example, calculation of Albania, 'all products binding coverage'.

$$
x_{1}^{*}(1)=\frac{\max x_{1}^{0}(1)-x_{1}^{0}(1)}{\max x_{1}^{0}(1)-\min x_{1}^{o}(1)}=\frac{100-100}{100-0}=0.0000
$$

Step 4: Obtained absolute values by calculating deviation sequence from desires value Equation 4

$$
\Delta_{0}(\gamma)=\left|x_{0}(\gamma)-x_{1}(\gamma)\right| \quad \text { Equation } 4
$$

\begin{tabular}{|c|c|c|c|c|c|c|c|}
\hline Sr. & Country & 1 & 2 & 3 & 4 & 5 & 6 \\
\hline $\mathbf{0}$ & Reference Sequence & 0.0000 & 0.0000 & 0.0000 & 0.0000 & 0.0000 & 0.0000 \\
\hline 1 & Albania & 1.0000 & 0.0429 & 0.0945 & 0.0000 & 0.0342 & 0.0300 \\
\hline 2 & Angola & 1.0000 & 0.4476 & 0.3025 & 0.0000 & 0.4737 & 0.3550 \\
\hline$\cdots$ & …........ & $\cdots$ & $\cdots$ & $\cdots$ & $\cdots$ & .. & $\cdots$ \\
\hline 100 & & & $\ddot{0}$ & 06022 & & $\ddot{30}$ & \\
\hline 109 & Pakistan & 0.9900 & 0.4762 & 0.6823 & 0.0000 & 0.2026 & 0.6000 \\
\hline 110 & Panama & 0.9400 & 0.2571 & 0.0086 & 0.0128 & 0.3684 & 0.2550 \\
\hline$\cdots$ & …........ & $\cdots$ & $\cdots$ & $\cdots$ & $\cdots$ & $\cdots$ & .. \\
\hline 100 & & 푸 & 02052 & $\ddot{3}$ & 씀ㅁ & $\ddot{0}$ & 푸요 \\
\hline $\begin{array}{l}157 \\
158\end{array}$ & Zambia & 0.1800 & 0.2952 & 0.3743 & 0.0000 & 0.1053 & 0.3700 \\
\hline 158 & Zimbabwe & 0.2400 & 0.2381 & 0.2610 & 0.7692 & 0.1447 & 0.2250 \\
\hline
\end{tabular}

Table 5: Deviation Sequences

For example, calculation of deviation for 'all products weighted mean tariff'.

$$
\Delta_{02}(2)=\left|x_{0}^{*}(2)-x_{2}^{*}(2)\right|=|1-0.5524|=0.4476
$$

Step 5: Established a co-efficient matrix of grey relation system Equation 5.

\begin{tabular}{|c|c|c|c|c|c|c|c|}
\hline Sr. & Country & 1 & 2 & 3 & 4 & 5 & 6 \\
\hline $\mathbf{0}$ & Reference Sequence & 1.0000 & 1.0000 & 1.0000 & 1.0000 & 1.0000 & 1.0000 \\
\hline 1 & Albania & 0.3333 & 0.9211 & 0.8411 & 1.0000 & 0.9360 & 0.9434 \\
\hline 2 & Angola & 0.3333 & 0.5276 & 0.6231 & 1.0000 & 0.5135 & 0.5848 \\
\hline ... & .......... & $\cdots$ & $\cdots$ & $\cdots$ & $\cdots$ & $\cdots$ & ... \\
\hline$\ldots$ & \#......... & $\ddot{3}$ & $0 \ddot{5122}$ & $0 \ddot{4220}$ & $\ddot{0} \ddot{0} 0$ & 07116 & 04545 \\
\hline $\begin{array}{l}109 \\
110\end{array}$ & $\begin{array}{l}\text { Pakistan } \\
\text { Panama }\end{array}$ & $\begin{array}{l}0.3356 \\
0.3472\end{array}$ & 0.6604 & $\begin{array}{l}0.4229 \\
0.9832\end{array}$ & 0.9750 & $\begin{array}{l}0.1110 \\
0.5758\end{array}$ & $\begin{array}{l}0.454 J \\
0.6623\end{array}$ \\
\hline ... & .......... & ... & $\cdots$ & $\cdots$ & $\cdots$ & $\cdots$ & ... \\
\hline$\ldots$ & $\ldots$ & & $\ldots$ & & & $\cdots$ & \\
\hline 157 & & 0.7353 & 0.6287 & 0.5719 & 1.0000 & 0.8261 & 0.5747 \\
\hline 158 & Zimbabwe & 0.6757 & 0.6774 & 0.6570 & 0.3939 & 0.7755 & 0.6897 \\
\hline
\end{tabular}

$$
\gamma\left[x_{0}^{*}(k), x_{i}^{*}(k)\right]=\frac{\Delta_{\min }+\xi \Delta_{\max }}{x_{0 i}(k)+\xi \Delta_{\max }}, 0<\gamma\left[x_{o}^{*}(k), x_{i}^{*}(k)\right] \leq 1 \quad \text { Equation } 5
$$

Table 6: Grey Relational Co-efficient

For example, grey relational co-efficient for 'all products weighted mean tariff'. 


$$
\gamma\left[x_{0}^{*}(2), x_{2}^{*}(2)\right]=\frac{\Delta_{\min +\xi} \Delta_{\max }}{\Delta_{2}(2)+\xi \Delta_{\max }}=\frac{0+(0.5) \times 1}{0.4476+(0.5) \times 1}=0.5276
$$

Step 6: Calculated GRA grade Equation 6 and Equation 7

$$
\begin{array}{cr}
\gamma\left(x_{0}^{*}, x_{i}^{*}\right)=\sum_{k=1}^{n} \beta_{k} \gamma\left[x_{0}^{*}(k), x_{i}^{*}(k)\right] & \text { Equation 6 } \\
\sum_{k=1}^{n} \beta_{k}=1 & \text { Equation } 7
\end{array}
$$

\begin{tabular}{|c|c|c|}
\hline Sr. & Country & GRGs \\
\hline $\mathbf{0}$ & Reference Sequence & 1.0000 \\
\hline 1 & Albania & 0.8291 \\
\hline 2 & Angola & 0.5971 \\
\hline$\cdots$ & ........... & $\cdots$ \\
\hline$\ldots$ & $\cdots \cdots \cdots \cdot$ & $\cdots$ \\
\hline 109 & Pakistan & 0.5728 \\
\hline 110 & Panama & 0.7006 \\
\hline$\cdots$ & $\cdots \cdots \cdots \cdot$ & $\cdots$ \\
\hline .. & n.......... & .. \\
\hline 157 & Zambia & 0.7228 \\
\hline 158 & Zimbabwe & 0.6449 \\
\hline
\end{tabular}

Table 7: Grey Relational Grades (GRGs)

For example, calculation of GRG for Angola:

$$
\begin{gathered}
\gamma\left(x_{0}^{*}, x_{2}^{*}\right)=\sum_{k=1}^{n} \beta_{k} \gamma\left[x_{0}^{*}(2), x_{2}^{*}(k)\right] \\
=0.1667 \times(0.3333+0.5276+0.6231+1.0000+0.5135+0.5848)=0.5971
\end{gathered}
$$

\section{Results \& Discussion}

The phenomenon of tariff barriers has of fundamental importance in international trade. It is an evergreen area investigated by economists. Therefore, the study in hand has also taken it as object of investigation. This study has approached the issue in different way. It used secondary data and applied mathematical methodology from gray system theory to analyze compositely a multitude of cross sections and criteria. Study used GRA, the results of which are given below (Table 8). In order to

\begin{tabular}{|c|c|c|c|c|c|c|c|c|}
\hline Country & GRG* & Rank & Country & GRG* & Rank & Country & GRG* & Rank \\
\hline $\begin{array}{l}\text { Reference } \\
\text { Sequence }\end{array}$ & 1.0000 & 0 & United Kingdom & 0.7770 & 27 & Ecuador & 0.6575 & 106 \\
\hline $\begin{array}{l}\text { Macao SAR, } \\
\text { China }\end{array}$ & 0.9402 & 1 & El Salvador & 0.7728 & 54 & Argentina & 0.6569 & 107 \\
\hline Hong Kong & 0.9167 & 2 & Indonesia & 0.7708 & 55 & Uruguay & 0.6555 & 108 \\
\hline Singapore & 0.8972 & 3 & North Macedonia & 0.7697 & 56 & Bangladesh & 0.6458 & 109 \\
\hline $\begin{array}{l}\text { Brunei } \\
\text { Darussalam }\end{array}$ & 0.8823 & 6 & Kyrgyz Republic & 0.7616 & 59 & Guyana & 0.6322 & 112 \\
\hline Myanmar & 0.8717 & 7 & Nicaragua & 0.7603 & 60 & Ghana & 0.6312 & 113 \\
\hline Chile & 0.8683 & 8 & Armenia & 0.7602 & 61 & Tonga & 0.6278 & 114 \\
\hline Peru & 0.8611 & 9 & Kazakhstan & 0.7557 & 62 & Mauritania & 0.6271 & 115 \\
\hline
\end{tabular}
provide logical and structured understanding to the readers the study has evolved scheme of classifying the countries on the basis of their performance on removing, eliminating or minimizing the tariff barriers.

Table 8: Results of Grey Relational Analysis 


\begin{tabular}{|c|c|c|c|c|c|c|c|c|}
\hline Albania & 0.8291 & 11 & Norway & 0.7459 & 64 & Cambodia & 0.6199 & 117 \\
\hline Lao PDR & 0.8218 & 12 & Switzerland & 0.7457 & 65 & Rwanda & 0.6164 & 118 \\
\hline Costa Rica & 0.8213 & 13 & Honduras & 0.7443 & 66 & Congo, Rep. & 0.6157 & 119 \\
\hline Eswatini & 0.8164 & 14 & Turkey & 0.7414 & 67 & Togo & 0.6150 & 120 \\
\hline Israel & 0.8161 & 15 & Papua New Guinea & 0.7403 & 68 & Cameroon & 0.6132 & 121 \\
\hline New Zealand & 0.8157 & 16 & Moldova & 0.7393 & 69 & Burkina Faso & 0.6126 & 122 \\
\hline Ukraine & 0.8153 & 17 & Japan & 0.7370 & 70 & Cote d'Ivoire & 0.6104 & 123 \\
\hline Oman & 0.8115 & 18 & Korea, Rep. & 0.7301 & 71 & Sri Lanka & 0.6082 & 124 \\
\hline Iceland & 0.8091 & 19 & Seychelles & 0.7271 & 72 & Dominica & 0.6082 & 125 \\
\hline Mexico & 0.8084 & 20 & Saudi Arabia & 0.7250 & 73 & Mali & 0.6024 & 126 \\
\hline Philippines & 0.8064 & 21 & Kuwait & 0.7245 & 74 & St. Vincent and Grenadines & 0.5996 & 127 \\
\hline Guatemala & 0.7983 & 22 & Colombia & 0.7244 & 75 & Gambia, The & 0.5985 & 128 \\
\hline Lesotho & 0.7944 & 23 & Qatar & 0.7229 & 76 & Angola & 0.5971 & 129 \\
\hline Botswana & 0.7941 & 24 & Zambia & 0.7228 & 77 & Trinidad and Tobago & 0.5927 & 130 \\
\hline Canada & 0.7930 & 25 & Malawi & 0.7190 & 78 & St. Lucia & 0.5921 & 131 \\
\hline Namibia & 0.7898 & 26 & China & 0.7187 & 79 & Vanuatu & 0.5920 & 132 \\
\hline Austria & 0.7770 & 27 & Vietnam & 0.7171 & 80 & Tunisia & 0.5894 & 133 \\
\hline Belgium & 0.7770 & 27 & Mongolia & 0.7167 & 81 & Guinea & 0.5862 & 134 \\
\hline Bulgaria & 0.7770 & 27 & Russian Federation & 0.7132 & 82 & Congo, Dem. Rep. & 0.5842 & 135 \\
\hline Croatia & 0.7770 & 27 & Thailand & 0.7098 & 83 & Venezuela, RB & 0.5837 & 136 \\
\hline Czech Republic & 0.7770 & 27 & Jordan & 0.7079 & 84 & Maldives & 0.5822 & 137 \\
\hline Denmark & 0.7770 & 27 & Morocco & 0.7076 & 85 & Samoa & 0.5750 & 138 \\
\hline Estonia & 0.7770 & 27 & Burundi & 0.7060 & 86 & Pakistan & 0.5728 & 139 \\
\hline Finland & 0.7770 & 27 & Paraguay & 0.7058 & 87 & Chad & 0.5709 & 140 \\
\hline France & 0.7770 & 27 & Malaysia & 0.7056 & 88 & Jamaica & 0.5656 & 141 \\
\hline Germany & 0.7770 & 27 & Yemen, Rep. & 0.7051 & 89 & Cabo Verde & 0.5648 & 142 \\
\hline Greece & 0.7770 & 27 & Panama & 0.7006 & 90 & Solomon Islands & 0.5621 & 143 \\
\hline Hungary & 0.7770 & 27 & Dominican Republic & 0.7001 & 91 & Niger & 0.5506 & 144 \\
\hline Ireland & 0.7770 & 27 & Madagascar & 0.6956 & 92 & Senegal & 0.5487 & 145 \\
\hline Italy & 0.7770 & 27 & South Africa & 0.6929 & 93 & Sierra Leone & 0.5466 & 146 \\
\hline Latvia & 0.7770 & 27 & Tajikistan & 0.6927 & 94 & Benin & 0.5438 & 147 \\
\hline Lithuania & 0.7770 & 27 & Uganda & 0.6863 & 95 & Grenada & 0.5430 & 148 \\
\hline Luxembourg & 0.7770 & 27 & Cuba & 0.6817 & 96 & Antigua and Barbuda & 0.5386 & 149 \\
\hline Malta & 0.7770 & 27 & United Arab Emirates & 0.6795 & 97 & Belize & 0.5368 & 150 \\
\hline Netherlands & 0.7770 & 27 & Suriname & 0.6717 & 98 & Central African Republic & 0.5326 & 151 \\
\hline Poland & 0.7770 & 27 & Tanzania & 0.6692 & 99 & Nepal & 0.5186 & 152 \\
\hline Portugal & 0.7770 & 27 & India & 0.6682 & 100 & Guinea-Bissau & 0.5166 & 153 \\
\hline Romania & 0.7770 & 27 & Nigeria & 0.6660 & 101 & Fiji & 0.5128 & 154 \\
\hline $\begin{array}{l}\text { Slovak } \\
\text { Republic }\end{array}$ & 0.7770 & 27 & Haiti & 0.6654 & 102 & Gabon & 0.5040 & 155 \\
\hline Slovenia & 0.7770 & 27 & Bahrain & 0.6651 & 103 & Barbados & 0.5017 & 156 \\
\hline Spain & 0.7770 & 27 & Bolivia & 0.6650 & 104 & Djibouti & 0.4886 & 157 \\
\hline Sweden & 0.7770 & 27 & Egypt, Arab Rep. & 0.6623 & 105 & St. Kitts and Nevis & 0.4810 & 158 \\
\hline
\end{tabular}

*GRG - Grey Relational Grade

Results of GRA show that Macao SAR China, Hong Kong, Singapore, Montenegro, Mauritius, Brunei Darussalam, Myanmar, Chile, Peru and Australia have relatively highest GRGs, therefore have relatively low tariff trade barriers, whereas, Grenada, Antigua and Barbuda, Belize, Central African Republic, Nepal, Guinea-Bissau, Fiji, Gabon, Barbados, Djibouti, St. Kitts and Nevis have lowest GRGs, therefore, have high level of tariff based barriers of international trade. All the member countries of European Union occupy rank 27 (i.e. all countries have the same rank) which can be explained in the perspective of their union on tariff. Since, they have uniformed tariff policy as against rest of the world, therefore, and have same GRG as for as the tariff barriers are concerned, therefore, occupy the same rank.

With an aim of assessment of world tariff barriers and analysis of secondary data is performed in order to rank the countries. It is vital to evaluate countries' performance qua each other. Applying the procedure of GRA the data was normalized, compared with reference series, deviation were calculated, GRA coefficient was worked out and the coefficient were computed. On the basis of GRG, grades have 
been computed and arranged in descending order. This methodology is different and unique and less applied in the area of economics. Hence, this study is different from contemporary studies; however its results are aligned with contemporary literature. The results of this study can be contrasted with that of some studies from existing literature (Table 9).

Table 9: Contrast of Results of the Study with Contemporary Literature

\begin{tabular}{|c|c|c|c|c|}
\hline Studies & Focus & Variables/Factors & Methodology & Results \\
\hline Current & Tariff barriers & $\begin{array}{l}\text { Weighted average mean of } \\
\text { tariff, products, tariff, share } \\
\text { of tariff, tariff rates. }\end{array}$ & $\begin{array}{l}\text { Grey relational } \\
\text { analysis }\end{array}$ & $\begin{array}{l}\text { China, Hong Kong and } \\
\text { Singapore rank on top of the } 158 \\
\text { countries having low tariff } \\
\text { barriers. EU occupy at rank } 27 . \\
\text { Gabon, Barbados, Djibouti and } \\
\text { Kitts \& Nevis occupy bottom, } \\
\text { hence, have high levels of tariff } \\
\text { trade barriers. }\end{array}$ \\
\hline $\begin{array}{l}\text { Kuenzel } \\
(2020)\end{array}$ & $\begin{array}{l}\text { WTO tariff } \\
\text { commitments and } \\
\text { temporary } \\
\text { protection }\end{array}$ & $\begin{array}{l}\text { Trade policy, political } \\
\text { economy motives, } \\
\text { macroeconomic factors, } \\
\text { import surges and terms-of- } \\
\text { trade effects, retaliation }\end{array}$ & $\begin{array}{l}\text { Fixed effects } \\
\text { logit model, } \\
\text { linear probability } \\
\text { model }\end{array}$ & $\begin{array}{l}\text { It is found a significant inverse } \\
\text { relationship between tariff } \\
\text { overhangs and trade policy } \\
\text { activity. }\end{array}$ \\
\hline $\begin{array}{l}\text { Lichtenberg } \\
\text { and Olson } \\
(2020)\end{array}$ & $\begin{array}{l}\text { Government- } \\
\text { induced production } \\
\text { commitment vs. } \\
\text { import tariff }\end{array}$ & $\begin{array}{l}\text { Production control policy, } \\
\text { domestic social welfare, } \\
\text { import tariff }\end{array}$ & $\begin{array}{l}\text { Empirical } \\
\text { analysis }\end{array}$ & $\begin{array}{l}\text { Different tariff lines share may } \\
\text { result in lower consumer welfare } \\
\text { and weaken United States trade } \\
\text { policy. }\end{array}$ \\
\hline $\begin{array}{l}\text { Suwanprasert } \\
\text { (2020) }\end{array}$ & $\begin{array}{l}\text { Trade } \\
\text { Restrictiveness } \\
\text { Index of Non- } \\
\text { Tariff Barriers }\end{array}$ & $\begin{array}{l}\text { Preference, production, non- } \\
\text { tariff barriers, equilibrium }\end{array}$ & $\begin{array}{l}\text { Simulation, } \\
\text { statistics } \\
\text { equation }\end{array}$ & $\begin{array}{l}\text { Aggregated of non-tariff barriers } \\
\text { can exactly be measured by } \\
\text { using proposed model. }\end{array}$ \\
\hline
\end{tabular}

The studies: Kuenzel (2020), Lichtenberg and Olson (2020) and Suwanprasert (2020) are comparable with the study in hand and are in general aligned with it. However, our study is different in number of countries under study, variables under study, methodology used, results and information contributed towards the body of knowledge.

\section{Conclusion}

International trade is a reality and the tariff barriers are intertwined with international trade. Rationalization, changes, elimination and reduction in tariff barriers is a continuous process having fundamental importance. It remained high on agenda of research in every regime. Comparing countries on basis of tariff is natural. This study also has same agenda but it approaches it in novel way. It uses GRA to compare countries and contributes valuable information in form of grey coefficients and grey relational grades. Study compares selected 158 countries on basis of six variables (Table 1). Results show that Macao SAR China, Hong Kong, Singapore, Montenegro, Mauritius, Brunei Darussalam, Myanmar, Chile, Peru and Australia are the countries that have high Grey Relational Grades (GRGs) rank on top among 158 countries meaning thereby low levels of tariff trade barriers. The member countries of EU (viz Austria, Belgium, Bulgaria, Croatia, Czech Republic, Denmark, Estonia, Finland, France, Germany, Greece, Hungary, Ireland, Italy, Latvia, Lithuania, Luxembourg, Malta, Netherlands, Poland, Portugal, Romania, Slovak Republic, Slovenia, Spain, Sweden and United Kingdom) have 0.7770 GRG and occupy rank 27 (i.e. the same rank). Since, EU is a union of European countries on tariff, therefore, they are graded identical qua rest of the world. Whereas, Grenada, Antigua and Barbuda, Belize, Central African Republic, Nepal, Guinea-Bissau, Fiji, Gabon, Barbados, Djibouti, St. Kitts and Nevis have low GRGs and occupy bottom ranks meaning thereby having high levels of tariff trade barriers. The study has theoretical and practical contributions towards body of knowledge that include grey relational coefficients and grades calculated for each country. It is useful for political governments for redressing policies, businessmen for rethinking and reframing the trade priorities, for researchers to refine the research frameworks. This study has some limitations as well. Firstly, it is based 
on cross sectional secondary data taken from WDI 2020 therefore the contextual limitation exists. Future studies may use some other dataset to verify the results qua reality. Secondly, it uses GRA as methodology the results should be confirmed through equivalent methods. Thirdly all variables have been given equal weights by dividing 1 by six (number of variables) this scheme can be rationalized by using Entropy method or expert opinion method.

\section{References}

Aisbett, E., \& Silberberger, M. (2020). Tariff liberalization and product standards: Regulatory chill and race to the bottom?. Regulation \& Governance.

Cambini, C., \& Soroush, G. (2019). Designing grid tariffs in the presence of distributed generation. Utilities Policy, 61, 100979.

Cary, M. (2020). Molecules of inefficiency: How tariffs impact carbon intensities, carbon dioxide emissions, and the environment. Science of The Total Environment, 713, 136531.

Chemingui, M. A., \& Dessus, S. (2008). Assessing non-tariff barriers in Syria. Journal of Policy Modeling, 30(5), 917-928.

Cheong, J., \& Tang, K. K. (2018). The trade effects of tariffs and non-tariff changes of preferential trade agreements. Economic Modelling, 70, 370-382.

Daly, M., Lizano, E., \& Silvy, A. (2000). Recent trends in tariff and nontariff barriers to trade in the United States. Review of World Economics, 136(4), 724-752.

DaSilva-Glasgow, D. (2020). Transactions Costs Perspective of Non-Tariff Barriers to Trade: An Analysis of Food and Agricultural Exports from Guyana Using Survey Data. The International Trade Journal, 34(3), 339-364.

Deng, X., Jing, R., \& Liang, Z. (2020). Trade liberalisation and domestic brands: Evidence from China's accession to the WTO. The World Economy, 43(8), 2237-2262.

Ertugrul, I., Oztas, T., Ozcil, A., \& Oztas, G. Z. (2016). Grey relational analysis approach in academic performance comparison of university: A case study of Turkish universities. European Scientific Journal, 7881, 128-139.

Estrades, C., \& Flores, M. (2020). Trade policymaking in Uruguay: Recent trends and challenges ahead. The World Economy, 43, 3133-3141.

Fugazza, M., \& Maur, J. C. (2008). Non-tariff barriers in CGE models: How useful for policy? Journal of Policy Modeling, 30(3), 475-490.

Giammetti, R. (2020). Tariffs, domestic import substitution and trade diversion in input-output production networks: An exercise on Brexit. Economic Systems Research, 1-33.

Grundke, R., \& Moser, C. (2019). Hidden protectionism? Evidence from non-tariff barriers to trade in the United States. Journal of International Economics, 117, 143-157.

Hayakawa, K., Ishikawa, J., \& Tarui, N. (2020). What goes around comes around: Export-enhancing effects of import-tariff reductions. Journal of International Economics, 126, 103362.

He, C., Li, C., \& Lin, C. (2019). Trade imbalance and the endogenous optimal tariff. Applied Economics, 51(10), 1040-1051.

Imbruno, M. (2016). China and WTO liberalization: Imports, tariffs and non-tariff barriers. China Economic Review, 38, 222-237.

Ino, H., \& Miyaoka, A. (2020). Government-induced production commitment vs. import-tariff under endogenous entry of foreign firms. The Journal of International Trade \& Economic Development, 124.

Jørgensen, J. G., \& Schröder, P. J. (2003). Reductions in real versus tariff barriers: the impact on industry concentration. Journal of Industry, Competition and Trade, 3(4), 251-268.

Juust, M., Vahter, P., \& Varblane, U. (2020). Trade Effects of the EU-South Korea Free Trade Agreement in the Automotive Industry. Journal of East-West Business, 1-29.

Kang, J. W., \& Dagli, S. (2018). Tariff barriers and industrial spillover effects. Journal of Korea Trade, 22(3), 228-246.

Knobel, A., Lipin, A., Malokostov, A., Tarr, D. G., \& Turdyeva, N. (2019). Deep integration in the 
Eurasian Economic Union: what are the benefits of successful implementation or wider liberalization?. Eurasian Geography and Economics, 60(2), 177-210.

Kuenzel, D. J. (2020). WTO tariff commitments and temporary protection: Complements or substitutes? European Economic Review, 121, 103344.

Lichtenberg, E., \& Olson, L. J. (2020). Tariffs and the risk of invasive pest introductions in commodity imports: Theory and empirical evidence. Journal of Environmental Economics and Management, 102321.

Liu, C., Lin, D., Liu, J., \& Li, Y. (2019). Quantifying the effects of non-tariff measures on African agrifood exporters. Agrekon, 58(4), 451-471.

Magee, S., Lee, H., \& Kim, J. (2019). Evidence and explanation for the Tariff-lobbying paradox: endogenous Tariffs fall as protectionist lobbying rises. Applied economics, 51(40), 4368-4384.

Maguire, K. (2001). Penetrating the Japanese market: The challenge of non- tariff barriers. European Business Review, 13(4), 241-250.

Manzoor, R., Maken, A. M., Syed, S. A., \& Ahmed, V. (2019). Trading with India: some current impediments for Pakistan. Journal of International Trade Law and Policy, 18(1), 39-55.

Nawaz, N. (2019). A dynamic model for an optimal specific import tariff. The International Trade Journal, 33(3), 255-276.

Niazi, A. A. K., Qazi, T. F., Basit, A.\&Shaukat, M. Z. (2021). Evaluation of Climate of Selected Sixtysix Countries using Grey Relational Analysis: Focus on Pakistan. Journal of Business and Social Review in Emerging Economies, 7(1), 51-62

Niu, Z., Liu, C., Gunessee, S., \& Milner, C. (2018). Non-tariff and overall protection: evidence across countries and over time. Review of World Economics, 154(4), 675-703.

Okumura, Y. (2015). Free trade networks on non-tariff barriers. Journal of Industry, Competition and Trade, 15(3), 223-238.

Ortiz Valverde, G., \& Latorre, M. C. (2020). A computable general equilibrium analysis of Brexit: Barriers to trade and immigration restrictions. The World Economy, 43(3), 705-728.

Qazi, T. F., Niazi, A. A. K., Asghar, W.\&Basit, A.(2021). Ease of Doing Business: Analysis of Trade Facilitations of One Hundred Twenty-Seven Countries of the World. Journal of Accounting and Finance in Emerging Economies, 7(1), 65-75.

Sampaio, P. R. P., \& Sampaio, R. S. R. (2020). The challenges of regulating water and sanitation tariffs under a three-level shared-authority federalism model: The case of Brazil. Utilities Policy, 64, 101049.

Santeramo, F. G., \& Lamonaca, E. (2019). The effects of non- tariff measures on agri- food trade: A review and meta- analysis of empirical evidence. Journal of Agricultural Economics, 70(3), 595617.

Schuenemann, F., \& Kerr, W. A. (2019). European Union non-tariff barriers to imports of African biofuels. Agrekon, 58(4), 407-425.

Slany, A. (2019). The role of trade policies in building regional value chains-some preliminary evidence from Africa. South African Journal of Economics, 87(3), 326-353.

Soon, B. M., \& Thompson, W. (2020). Non-tariff barrier on chicken imports into Russia: Impact on production, trade and prices. Journal of Policy Modeling, 42(3), 583-596.

Suwanprasert, W. (2020). Trade Restrictiveness Index of Non-Tariff Barriers Under the CES Preference. International Economic Journal, 34(1), 48-57.

Toshimitsu, T. (2008). On the effects of emission standards as a non-tariff barrier to trade in the case of a foreign Bertrand duopoly: A note. Resource and Energy Economics, 30(4), 578-584.

Tovar, P. (2019). Preferential and multilateral liberalization: Evidence from Latin America's use of tariffs, antidumping and safeguards. Journal of Development Economics, 141, 102383.

Ural Marchand, B. (2019). Inequality and trade policy: The pro- poor bias of contemporary trade restrictions. Review of Income and Wealth, 65, S123-S152.

Vadila, Y., \& Resosudarmo, B. P. (2020). Tariff reform and income inequality in I ndonesia. Regional Science Policy \& Practice, 12(3), 455-475. 
Winchester, N. (2009). Is there a dirty little secret? Non-tariff barriers and the gains from trade. Journal of policy modeling, 31(6), 819-834.

World Development Indicators. (2020). Retrieved April 15, 2020, from http://wdi.worldbank.org/tables

Zhang, X., Haviarova, E., \& Zhou, M. (2020). A welfare analysis of China's tariffs on US hardwood products. Forest Policy and Economics, 113, 102085. 